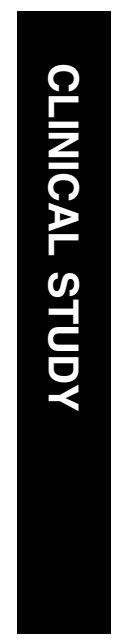

\title{
Treatment outcomes for primary intraocular lymphoma: implications for external beam radiotherapy
}

A Berenbom¹, RM Davila², H-S Lin ${ }^{3}$ and JW Harbour $^{1}$ may still be the most appropriate first-line treatment in most cases.

Eye (2007) 21, 1198-1201; doi:10.1038/sj.eye.6702437; published online 26 May 2006

Keywords: intraocular lymphoma; CNS lymphoma; radiotherapy; chemotherapy

\section{Introduction}

Primary intraocular lymphoma (PIOL) is often associated with central nervous system (CNS) involvement and is often fatal. External beam radiotherapy, with or without adjuvant systemic chemotherapy, has been the mainstay of ocular treatment for many years. ${ }^{1}$ Recently, intravitreal injection of chemotherapeutic agents such as methotrexate has gained popularity as an alternative treatment for intraocular disease, largely fuelled by the perception that radiotherapy conveys a high risk of tumour recurrence and severe complications. ${ }^{2-4}$ Using contemporary radiotherapeutic techniques, however, we have not appreciated such dismal outcomes. Additionally, intravitreal chemotherapy requires repetitive injections and can be associated with serious complications such as cataracts, corneal epitheliopathy, maculopathy, vitreous haemorrhage, optic atrophy, retinal detachment, and endophthalmitis. ${ }^{3}$ Thus, the purported drawbacks of radiotherapy need to be weighed against the risks of intravitreal injections. As the rarity of PIOL prohibits a prospective clinical trial to compare these two treatment approaches, we have analysed our treatment outcomes over the last decade to clarify the efficacy and complications of radiotherapy.
Abstract

Purpose Intravitreal chemotherapy for primary intraocular lymphoma (PIOL) increasingly is promoted as an alternative to \author{
radiotherapy, owing to putative high failure \\ and complication rates of the latter modality. \\ Our aim was to confirm whether these \\ concerns about radiotherapy were borne out in \\ patients treated at our institution over the last \\ decade. \\ Design Retrospective interventional case \\ series. \\ Participants A total of 21 eyes of 12 patients \\ with PIOL. \\ Methods Comprehensive chart review of \\ ophthalmologic and systemic manifestations, \\ treatments, and outcomes. \\ Main outcome measures Radiation \\ complications and local tumour control. \\ Results Cytology-confirmed lymphoma \\ involved one eye in three patients and both \\ eyes in nine patients. Initial treatment \\ included external beam radiotherapy and \\ chemotherapy (six patients), chemotherapy \\ alone (four patients), radiotherapy alone (one \\ patient), and no treatment (one patient). \\ Ocular relapses occurred in no patients \\ receiving radiotherapy and in two patients \\ who did not receive radiotherapy. \\ Complications of radiotherapy included dry \\ eye (four patients), cataract (four patients), and \\ mild radiation retinopathy (two patients). \\ Conclusions Radiotherapy for PIOL is highly \\ effective with acceptable complications. In the \\ absence of a clear advantage to intravitreal \\ chemotherapy, which involves repetitive \\ injections and associated risks, radiotherapy \\ 21 April 2006 \\ Published online: 26 May \\ 2006 \\ wustl.edu \\ Received: 19 January 2006 \\ Correspondence: \\ Harbour, \\ Ophthalmology and Visual \\ Washington University \\ Campus Box 8096, 66 \\ Tel: + 1314747 1738; \\ E-mail: harbour@
}

. 


\section{Method}

Institutional Review Board approval was obtained. Patients with cytology-proven PIOL were included if they were evaluated between January 1995 and July 2003. Patients were excluded if the clinical diagnosis was not confirmed by pathologic diagnosis or if there was evidence of a systemic lymphoma not located in the CNS, in which case the intraocular lymphoma was assumed to represent metastatic disease. Twelve patients met these criteria. Our method for obtaining a cytologic specimen at vitrectomy previously has been described. ${ }^{5}$ Data recorded for each patient included patient age, gender, ethnicity, involved eye(s), visual acuity, presenting clinical signs, characteristics on ultrasound and fluorescein angiography, systemic evaluation, results of computed tomography, magnetic resonance imaging, bone marrow biopsy and lumbar puncture, presence of CNS lymphoma at diagnosis or thereafter, diagnostic method and results, types and dates of treatments, radiation dose and dose rate, chemotherapeutic agents, treatment complications, recurrences, date of last follow-up, and ocular and systemic status at last follow-up. Comparison of continuous variables was performed using Student's $t$-test. Time-dependent variables were analysed by Kaplan-Meier life table analysis using MedCalc software, version 7.2.0.2.

\section{Results}

Twenty-one eyes of 12 immunocompetent patients met inclusion criteria for the study (Table 1). The average age was 60 years with a range of 36-75. Four patients were male, and eight patients were female. Ethnic

backgrounds included Caucasian (nine patients), Indian (one patient), Middle Eastern (one patient), and Japanese (one patient). None of the patients were immunocompromized. CNS lymphoma was diagnosed 31 months before ocular diagnosis in one patient, concurrent with ocular diagnosis in four patients, and subsequent to ocular diagnosis in six patients. At the time of ocular diagnosis, six patients had bilateral ocular disease with no CNS lymphoma, one patient had unilateral disease with no CNS lymphoma, two patients had unilateral disease with CNS involvement, and three patients had bilateral disease with CNS involvement. Presenting ocular signs included vitreous cells (21 eyes), anterior segment cells (nine eyes), subretinal infiltrates (six eyes) and acute retinal necrosis (one eye).

Systemic work-up included cranial magnetic resonance imaging (11 patients), lumbar puncture (10 patients), bone marrow biopsy (six patients), and chest and abdominal computed tomography (five patients). Magnetic resonance imaging disclosed CNS disease in

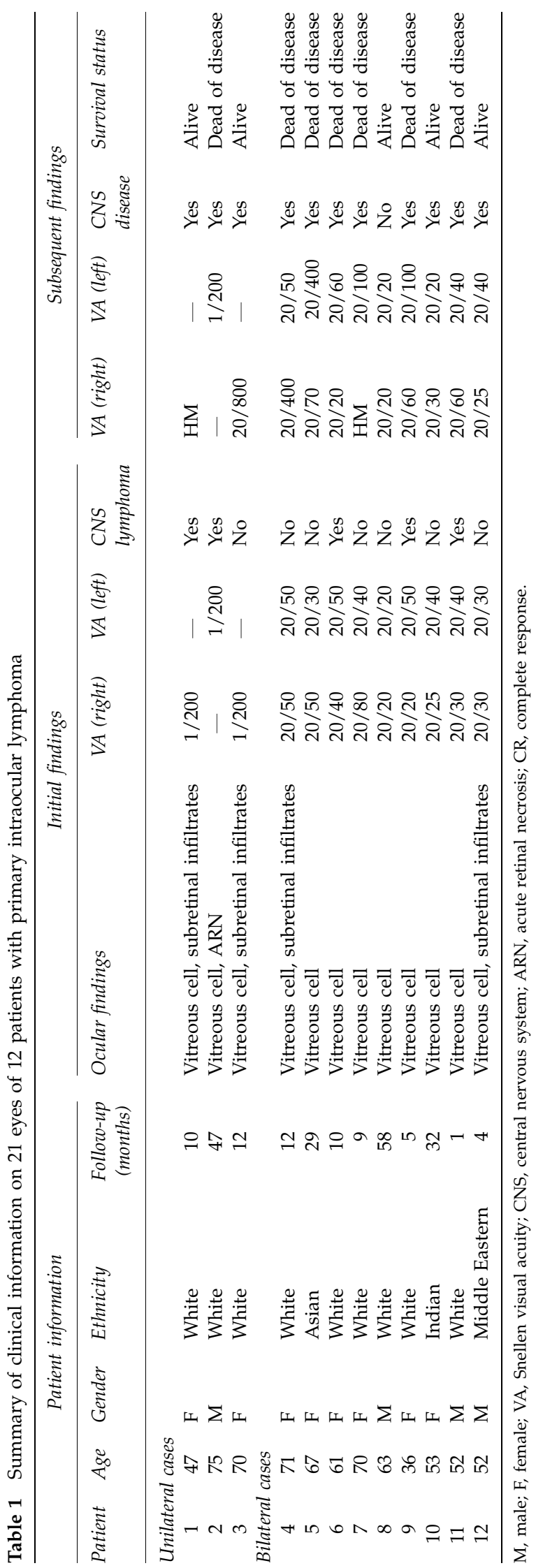


Table 2 Treatment outcomes for patients with primary intraocular lymphoma

\begin{tabular}{|c|c|c|c|c|c|}
\hline Patient & $\begin{array}{l}\text { Initial ocular } \\
\text { radiotherapy }\end{array}$ & $\begin{array}{l}\text { Initial brain } \\
\text { radiotherapy }\end{array}$ & $\begin{array}{l}\text { Systemic } \\
\text { chemotherapy }^{\text {a }}\end{array}$ & $\begin{array}{l}\text { Intraocular } \\
\text { recurrence }\end{array}$ & Complications \\
\hline 1 & Yes & Yes & $\mathrm{M}, \mathrm{V}, \mathrm{R}, \mathrm{P}, \mathrm{A}$ & No & Dry eye \\
\hline 2 & None & No & None & - & - \\
\hline 3 & Yes & No & $\mathrm{M}, \mathrm{V}, \mathrm{R}, \mathrm{P}$ & No & Dry eye \\
\hline 4 & None & No & NK & NK & None \\
\hline 5 & Yes & Yes & $\mathrm{D}, \mathrm{C}, \mathrm{A}$ & No & Cataract, retinopathy \\
\hline 6 & Yes & Yes & $\mathrm{D}, \mathrm{C}, \mathrm{A}$ & No & Dry eye \\
\hline 7 & None & Yes & M & No & None \\
\hline 8 & Yes & No & $\mathrm{M}, \mathrm{V}, \mathrm{R}, \mathrm{P}, \mathrm{A}$ & No & Retinopathy \\
\hline 9 & Yes & No & Yes $^{\mathrm{b}}$ & No & None \\
\hline 10 & None $^{c}$ & No & $\mathrm{M}, \mathrm{V}, \mathrm{R}, \mathrm{P}, \mathrm{A}$ & Yes & Cataract \\
\hline 11 & None & Yes & Yes $^{\mathrm{b}}$ & Yes & - \\
\hline 12 & Yes & No & None & No & Cataract, dry eye \\
\hline
\end{tabular}

${ }^{\mathrm{a}} \mathrm{A}$, ara-C; C, carboplatin; D, dexamethasone; M, methotrexate; $\mathrm{P}$, procarbazine; $\mathrm{R}$, rituxan; $\mathrm{V}$, vincristine; NK, not known.

${ }^{b}$ Chemotherapy regimen not known.

'Both eyes were treated with external beam radiotherapy after chemotherapy alone led to intraocular recurrence.

four patients. Lumbar puncture revealed atypical lymphoid cells in the cerebrospinal fluid of three patients, all of whom later developed CNS disease. Bone marrow biopsy was negative in all cases. Computed tomography showed no evidence of visceral lymphoma in any patients. The diagnosis of large-cell lymphoma was made by cytologic evaluation of vitreous material obtained by pars plana vitrectomy in all 12 patients.

Ocular and systemic treatments are summarized in Table 2. Initial ocular treatment included external beam radiotherapy and chemotherapy (six patients), chemotherapy alone (four patients), radiotherapy alone (one patient), and no treatment owing to poor systemic status (one patient). Most ocular radiotherapy was delivered in 15 fractions at 2 Gy per fraction for a total of 30-35 Gy using a pair of parallel opposed portals and six MV X-rays. Chemotherapeutic regimens included intravenous methotrexate, vincristine, rituxan, and procarbazine (four patients), dexamethasone and carboplatin (two patients), high-dose methotrexate alone (one patient), and an unknown regimen (three patients). Five patients were consolidated with ara-C, and one patient also received intrathecal methotrexate.

Ocular recurrence was detected in no patients receiving radiotherapy and in two patients not receiving radiotherapy. Patient No. 10 underwent chemotherapy as the initial treatment for intraocular lymphoma and 14 months later developed recurrence in both eyes that responded to radiotherapy. Patient \#11 received chemotherapy for CNS lymphoma and 31 months later developed intraocular lymphoma. Radiation complications included dry eye in four patients, cataract in four patients, and mild radiation retinopathy in two patients.

Of the seven patients without CNS involvement at ocular diagnosis, six patients later developed CNS disease at a mean time of 16.3 months from ocular diagnosis. For these six patients, the mean time from ocular diagnosis to detection of CNS disease was 28 months for those who received chemotherapy, compared to 8 months for those who did not receive chemotherapy $(P=0.24)$. After mean follow-up of 19 months (range, 158 months), seven patients had died of CNS disease, four patients were alive with CNS disease, and one patient was alive without CNS disease.

\section{Discussion}

In our experience with PIOL over the last decade, radiotherapy has resulted in excellent local tumour control and few complications. The only two patients who experienced intraocular recurrence were treated initially with systemic chemotherapy rather than radiotherapy. Thus, our results draw into question previous studies suggesting a high local recurrence rate with radiotherapy. ${ }^{3}$ There may be several explanations for this discrepancy. First, it is possible that our patients later will develop recurrence, although our follow-up was similar to other studies. ${ }^{3}$ Second, previous authors, such as Smith et al, ${ }^{3}$ have cited results of radiotherapy with CNS lymphoma, ${ }^{6}$ as evidence for the ineffectiveness of radiotherapy. However, we do not believe that it is appropriate to extrapolate the outcomes in CNS lymphoma to PIOL, because PIOL is more radiosensitive and has more favourable dosimetric constraints than CNS lymphoma. Third, our results may reflect improvements in radiotherapy techniques compared to those used in older studies.

The complication rates observed in our study likewise were superior to previous reports. Smith et $a l^{3}$ suggested that radiotherapy for PIOL carries a high risk of neurologic toxicity, severe radiation retinopathy, and 
irreversible visual loss. However, we did not observe any of these severe complications in our series. Only two of our patients developed radiation retinopathy, and one retained 20/20 visual acuity while the other retained 20/70 acuity in the better eye. The most common complications that we encountered were dry eyes, which were controlled with topical lubricants, and cataracts, which were amenable to standard cataract extraction. These favourable results were in spite of the fact that most patients received systemic chemotherapy, which increases the risk of radiation retinopathy. The lower complication rates in this study are likely owing to the lower radiation doses and improved dosimetric techniques that have been employed over the last decade. PIOL has been treated in some centres with high total radiation doses - often over $50 \mathrm{~Gy}$ - similar to those used for CNS lymphoma. ${ }^{7-9}$ However, PIOL is now recognized to be more radiosensitive than $\mathrm{CNS}$ lymphoma and can be treated with significantly lower radiation doses. Typically, we use 35-40 Gy given in 15 fractions to both globes, and customized blocks are made following computed tomographic simulation to minimize normal tissue toxicity.

Although this is a small, retrospective, uncontrolled series with limited follow-up, it draws into question the purported inefficacy and severe complications associated with radiotherapy for PIOL. The relatively mild complications observed in our study compared favourably with the cataracts, corneal epitheliopathy, maculopathy, vitreous haemorrhage, optic atrophy, retinal detachment, and endophthalmitis that have been associated with intravitreal chemotherapy. ${ }^{3}$ As no current treatments are curative, and most patients eventually succumb to CNS recurrence, ${ }^{10}$ a major goal of ocular treatment is to maximize the quality of life. Radiotherapy is well tolerated by most patients, whereas intravitreal chemotherapy requires repetitive injections that can be uncomfortable. When taken together with excellent local control rates and acceptable complications with radiotherapy, we do not see an advantage to using intravitreal chemotherapy as a first-line therapy for PIOL.

Currently, we recommend external beam radiotherapy to both eyes as the initial treatment for intraocular lymphoma. The decisions whether to include the CNS in the radiation field and whether to administer systemic chemotherapy will depend on CNS status and other factors and are made in consultation with the medical oncologist. Intrathecal chemotherapy and autologous stem cell transplantation are usually reserved for recurrent or refractory CNS disease. , $^{31,12}$ We view intravitreal methotrexate as a second-line therapy for patients who fail radiotherapy, and for patients who are at high risk for radiation retinopathy, such as those with previous ocular radiation exposure and those with significant diabetic retinopathy.

\section{Conclusion}

Ocular external beam radiotherapy is highly effective in controlling PIOL and is associated with acceptable ocular complications.

\section{References}

1 Harbour JW, Char DH. Intraocular lymphoid tumors. In: Chang S, Shields J, Green WR (eds). Retina, Vitreous, Macular: A Comprehensive Text. WB Saunders: Philadelphia, 1998, p 1204-1216.

2 Fishburne BC, Wilson DJ, Rosenbaum JT, Neuwelt EA. Intravitreal methotrexate as an adjunctive treatment of intraocular lymphoma. Arch Ophthalmol 1997; 115: 1152-1156.

3 Smith JR, Rosenbaum JT, Wilson DJ, Doolittle ND, Siegal T, Neuwelt EA et al. Role of intravitreal methotrexate in the management of primary central nervous system lymphoma with ocular involvement. Ophthalmology 2002; 109: 1709-1716.

4 Jahnke K, Bechrakis NE, Coupland SE, Schmittel A, Foerster $\mathrm{MH}$, Fischer $\mathrm{L}$ et al. Treatment of primary intraocular lymphoma with oral trofosfamide: report of two cases and review of the literature. Graefes Arch Clin Exp Ophthalmol 2004; 242: 771-776.

5 Farkas T, Harbour JW, Davila RM. Cytologic diagnosis of intraocular lymphoma in vitreous aspirates. Acta Cytol 2004; 48: 487-491.

6 Schlegel U, Schmidt-Wolf IG, Deckert M. Primary CNS lymphoma: clinical presentation, pathological classification, molecular pathogenesis and treatment. J Neurol Sci 2000; 181: 1-12.

7 Abrey LE, DeAngelis LM, Yahalom J. Long-term survival in primary CNS lymphoma. J Clin Oncol 1998; 16: 859-863.

8 Laack NN, Brown PD. Cognitive sequelae of brain radiation in adults. Semin Oncol 2004; 31: 702-713.

9 Hoffman PM, McKelvie P, Hall AJ, Stawell RJ, Santamaria JD. Intraocular lymphoma: a series of 14 patients with clinicopathological features and treatment outcomes. Eye 2003; 17: 513-521.

10 Freeman LN, Schachat AP, Knox DL, Michels RG, Green WR. Clinical features, laboratory investigations, and survival in ocular reticulum cell sarcoma. Ophthalmology 1987; 94: 1631-1639.

11 Char DH, Ljung BM, Miller T, Phillips T. Primary intraocular lymphoma (ocular reticulum cell sarcoma) diagnosis and management. Ophthalmology 1988; 95: 625-630.

12 Chan CC, Wallace DJ. Intraocular lymphoma: update on diagnosis and management. Cancer Control 2004; 11: 285-295. 